\title{
Calculation of higher order corrections to positronium energy levels
}

\section{Gregory S. Adkins*}

Franklin \& Marshall College, Lancaster, Pennsylvania 17604, USA

E-mail: gadkinsefandm.edu

\section{Benjamin Akers}

Department of Physics, Northeastern University, Boston, Massachusetts 02115, USA

E-mail: b.akers@northeastern.edu

\section{Md Faisal Alam}

Department of Physics, University of Illinois at Urbana-Champaign, Urbana, Illinois 61801, USA

E-mail: mfalam2@illinois.edu

\section{Lam M. Tran}

Department of Physics, The Pennsylvania State University, University Park, Pennsylvania 16802, USA

E-mail: lammtran@outlook.com

\section{Xuan Zhang}

Facebook, Menlo Park, California 94025, USA

E-mail: xuan.zhang707@gmail.com

\begin{abstract}
We report on progress in the calculation of corrections to positronium energy levels of order $m \alpha^{7}$. Corrections at this level will be needed for the interpretation of the results of upcoming measurements. A procedure for the calculation of high order corrections has been developed based on the Bethe-Salpeter equation of dimensionally regularized NRQED and the method of regions. We demonstrate the effectiveness of this approach by using it to obtain all pure recoil corrections to positronium energies at $O\left(m \alpha^{6}\right)$ in a unified manner.
\end{abstract}

International Conference on Precision Physics and Fundamental Physical Constants - FFK2019

9-14 June, 2019

Tihany, Hungary

${ }^{*}$ Speaker. 


\section{Introduction}

Positronium stands out as a particularly simple nonrelativistic two-body bound system. The electron and positron in positronium have no known substructure, and the interaction between them is due purely to QED to an excellent approximation. Positronium is subject to large "recoil" effects-its mass ratio takes the maximum possible value of $m_{e^{-}} / m_{e^{+}}=1$. Because positronium is a particle-antiparticle bound system it is subject to annihilation-real and virtual-and its states can be taken to be eigenstates of both parity and charge conjugation. Positronium is accessible to high-precision measurements of energy levels and decay rates, so calculation of these quantities to similar or greater precision allows for a stringent test of the methods of calculation and the assumptions upon which those calculations are based.

Positronium has been the subject of much work, both experimental and theoretical, since and even before its discovery by Deutsch in 1951 [1]. Measurements of steadily increasing precision were preformed for the decay rates of the $n=1$ triplet and singlet states and for transition energies involving the various $n=1$ and $n=2$ states. Reviews of this work appear as [2-7]. Positronium energy levels can be expressed as the electron mass $m$ times a series in the fine structure constant $\alpha$ and logarithms of $\alpha$ :

$$
\begin{aligned}
E=m & \left\{A_{20} \alpha^{2}+A_{40} \alpha^{4}+A_{51} \alpha^{5} \ln (1 / \alpha)+A_{50} \alpha^{5}+A_{61} \alpha^{6} \ln (1 / \alpha)+A_{60} \alpha^{6}\right. \\
& \left.+A_{72} \alpha^{7} \ln ^{2}(1 / \alpha)+A_{71} \alpha^{7} \ln (1 / \alpha)+A_{70} \alpha^{7}+\cdots\right\}
\end{aligned}
$$

where we are using natural units with $\hbar=c=1$. The contributions at $O\left(m \alpha^{2}\right)$ are just the Bohr energies with reduced mass. The terms at $O\left(m \alpha^{4}\right)$ represent the fine and hyperfine structure corrections (which have the same order for positronium because the masses are equal), and the $m \alpha^{5}$ effects are further corrections including the Lamb shift and Salpeter term. These contributions are discussed in the reviews listed above. Many authors contributed to the calculation at order $m \alpha^{6}$, which was completed by the year $2000[8,9]$ for $\mathrm{S}$ and P states, and by 2008 for states with $\ell>1[10,11]$. The leading $\log$ corrections at order $m \alpha^{7}$ were done by 2000 as well [12-14], and the $O\left(m \alpha^{7} \ln (1 / \alpha)\right)$ corrections to the hyperfine splitting $\left(1^{3} S_{1}-1{ }^{1} S_{0}\right)$ appeared shortly thereafter [15-17]. Only a relative few of the pure $O\left(m \alpha^{7}\right)$ corrections have been completed as yet. The ones that have been done involve "ultrasoft" corrections [18], "hard" (i.e. high-energy) corrections in the two-photon-exchange channel [19-23], corrections in the three-photon-annihilation channel [24], corrections in the two-photon-annihilation channel [25-28], and corrections in the one-photon-annihilation channel [29].

Our overall project is to complete the calculation of all contributions at $O\left(m \alpha^{7}\right)$. We are starting with the "pure recoil" corrections-that is, corrections without radiative photons (radiative photons are those connected to just the electron or just the positron) or annihilation photons, and that involve equal powers of the electron charge and positron charge. The pure recoil corrections provide the deepest probe of the binding process. We have developed a method of calculation involving dimensionally regularized NRQED and the NRQED Bethe-Salpeter equation, described in more detail in [30] and in Sect. 2. We are using this approach to complete a unified calculation of all corrections to positronium energy levels up to order $m \alpha^{7}$. So far, we have been able to reproduce all contributions at $O\left(m \alpha^{4}\right), O\left(m \alpha^{5}\right)$, and $O\left(m \alpha^{6}\right)$, finding the traditional results as 
given, for instance, in [31] at $O\left(m \alpha^{4}\right)$ and [32] at $O\left(m \alpha^{5}\right)$. Our results at $O\left(m \alpha^{6}\right)$ are given and discussed in Sect. 3.

\section{Method of calculation}

In this section we give a brief description of our method of calculation. More details were given in [30]. We will give a complete account in a future publication.

Our calculation is based on the effective low-energy quantum field theory Nonrelativistic QED (NRQED) [33] in Coulomb gauge. NRQED is applicable to low energy processes (involving energies and momenta small compared to the electron rest energy $m$ ), such as binding in atoms like positronium. In its domain of applicability it is equal in rigor to QED, but it is optimized for the calculation of effects involving nonrelativistic energies. Relativistic corrections are encoded in a number of additional interactions making it relatively easy to keep track of the order in $\alpha$ of various corrections. Detailed forms for the NRQED effective Lagrangian and Feynman rules have been given in a number of places $[34,35]$. All divergences-ultraviolet and infrared-are regulated using dimensional regularization.

We use the NRQED Bethe-Salpeter equation to find a description of the positronium system. A perturbative scheme for finding energy levels is built upon an exactly soluble lowest order description in which the interaction kernel is simply the nonrelativistic Coulomb interaction (in $D=3-2 \varepsilon$ spatial dimensions). The lowest order Bethe-Salpeter equation is just the usual nonrelativistic Schrödinger equation with known solutions [36, 37]. Feynman diagrams for the perturbations, such as those shown in Fig. 1, are treated as follows. First, we performed all energy integrals by use of the residue theorem. The integrals that remained were over the spatial momenta of both external wave functions and internal loops. We identified two regions for the various spatial momenta occurring in these integrals [38]: soft (of order $m \alpha$ ) and ultrasoft (of order $m \alpha^{2}$ ). With assumptions about the order in $\alpha$ of each momentum, we expanded in powers of $\alpha$ to obtain a number of terms having well defined orders. We were able to find analytic results for all contributions at $O\left(m \alpha^{6}\right)$ as detailed in the following section.

\section{Results at order $m \alpha^{6}$}

The graphs that contribute $m \alpha^{6}$ corrections are shown in Fig. 1 . These were evaluated one by one using the approach outlined in Sec. 2. They were evaluated in a unified way for both spin states, all orbital angular momenta, and all $n$ by identifying contributions at the order of interest, reducing them to ordinary Coulomb expectation values (albeit in $D=3-2 \varepsilon$ dimensions), and evaluating the expectation values as outlined in [37]. We give our results separately for $\ell=0$ and $\ell>0$ since only for $\ell=0$ were there cancelling divergences from different scales that appeared at intermediate stages of the calculation along with the associated logarithms. For the hyperfine splitting $\Delta E_{\mathrm{hfs}}^{\ell=0}=\Delta E^{\ell=0, s=1}-\Delta E^{\ell=0, s=0}$ we find

$$
\Delta E_{\mathrm{hfs}}^{\ell=0}=\frac{m \alpha^{6}}{n^{3}}\left\{-\frac{1}{6} \ln \left(\frac{\alpha}{n}\right)-\frac{1}{6} H_{n}-\frac{1}{2 n^{2}}+\frac{3}{4 n}+\frac{295}{432}-\frac{\ln 2}{4}+\frac{5}{12 \pi^{2}}-\frac{17 \zeta(3)}{8 \pi^{2}}\right\},
$$




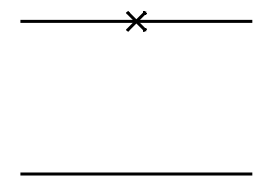

(a)

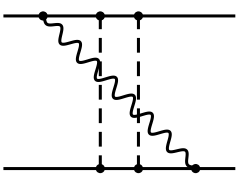

(f)

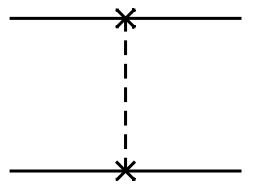

(b)

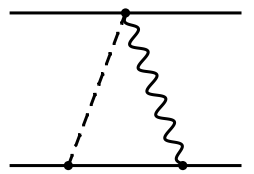

(g)

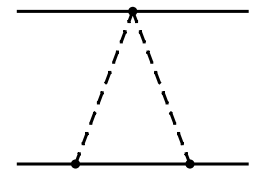

(c)

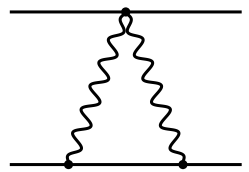

(h)

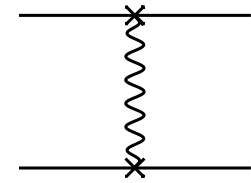

(d)

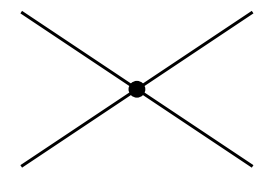

(i)

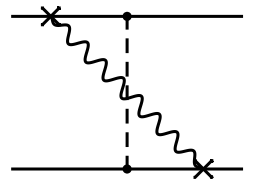

(e)

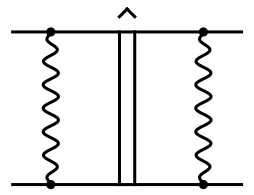

(j)

Figure 1: NRQED kernels that contribute recoil corrections to the bound state energy levels through order $m \alpha^{6}$. Additional diagrams reflected vertically or horizontally also contribute. Term (a) represents the sixthorder relativistic kinetic energy correction. There are two such diagrams, with the correction on the electron line (top) or positron line (bottom). Term (b) represents all corrections involving the exchange of a single Coulomb photon with Darwin or spin-orbit corrections on each end, or with a Coulomb vertex on one end and a higher-order vertex on the other. Term (c) shows a " $\Lambda$ " graph involving Coulomb photons having a Coulomb-Coulomb seagull on the top and two Coulomb vertices on the bottom. The vertically reflected graph is also included. Term (d) represents a transverse photon with a convection, Fermi, or higher-order vertex on each end. Term (e) shows crossed transverse and Coulomb photons with various vertices for the transverse photon. Term (f) has a transverse photons with convection or Fermi vertices crossing two Coulomb photons. Term (g) is a Coulomb-transverse " $\Lambda$ " graph. Term (h) is a transverse-transverse " $\Lambda$ " graph. Term (i) is a four-fermion "contact" term. This graph contains the effects of the six hard threephoton-exchange graphs. Term (j) represents the contribution from second-order perturbation theory. The "photons" shown here really represent the fourth-order interaction coming from first corrections to Coulomb photon exchange and the lowest order contributions to transverse photon exchange, while the object in the center represents the Coulomb Green function with the pole for the state in question subtracted out.

where $H_{n}=\sum_{n=1}^{\infty} \frac{1}{n}$ is the $n^{\text {th }}$ harmonic number, in agreement with the results of $[9,39,40]$. For the spin average $\Delta E_{\text {avg }}^{\ell=0}=\frac{1}{4}\left(3 \Delta E^{\ell=0, s=1}+\Delta E^{\ell=0, s=0}\right)$ we obtain

$$
\Delta E_{\mathrm{avg}}^{\ell=0}=\frac{m \alpha^{6}}{n^{3}}\left\{-\frac{69}{512 n^{3}}+\frac{1}{3 n^{2}}-\frac{1}{4 n}-\frac{83}{576}-\frac{11}{16 \pi^{2}}-\frac{3 \zeta(3)}{8 \pi^{2}}\right\}
$$

in agreement with the results of $[9,41]$. We performed an independent calculation of the hard threephoton-exchange graphs that give rise to the contact term matching coefficient of Fig. 1i using the integration by parts routine FIRE [42]. For the second order perturbation theory contribution of Fig. $1 \mathrm{j}$ we made extensive use of formulas for the reduced Coulomb Green function contained in [43]. New angular momentum structures appeared in the calculation of corrections with $\ell>0$. For a given $n$ and $\ell$ there are four states, having $\{s, j\}=\{0, \ell\},\{1, \ell-1\},\{1, \ell\}$, and $\{1, \ell+1\}$. We found it convenient to express our results in terms of four independent angular momentum structures: $1, \vec{S}^{2}=s(s+1),\langle\vec{L} \cdot \vec{S}\rangle=\frac{1}{2}\left\{\vec{J}^{2}-\vec{L}^{2}-\vec{S}^{2}\right\}$, and $\xi(j, \ell, s)=\frac{2 \vec{L}^{2} \vec{S}^{2}-3 \vec{L} \cdot \vec{S}(2 \vec{L} \cdot \vec{S}+1)}{3\left(4 \vec{L}^{2}-3\right)}$. The 
total result for energy levels with $\ell>0$ is

$$
\begin{aligned}
\Delta E^{\ell>0}=m \alpha^{6}\{ & {\left[-\frac{3\left(-33+68 \ell+68 \ell^{2}\right)}{64 \ell(1+\ell)(-1+2 \ell)(1+2 \ell)(3+2 \ell) n^{5}}+\frac{9\left(-3-13 \ell+15 \ell^{2}+56 \ell^{3}+28 \ell^{4}\right)}{32 \ell^{2}(1+\ell)^{2}(-1+2 \ell)(1+2 \ell)^{2}(3+2 \ell) n^{4}}\right.} \\
& \left.+\frac{3\left(9+54 \ell-39 \ell^{2}-850 \ell^{3}-1077 \ell^{4}+2040 \ell^{5}+5384 \ell^{6}+4032 \ell^{7}+1008 \ell^{8}\right)}{32 \ell^{3}(1+\ell)^{3}(-1+2 \ell)^{2}(1+2 \ell)^{3}(3+2 \ell)^{2} n^{3}}\right] \xi(j, \ell, s) \\
+ & {\left[\frac{99-140 \ell-140 \ell^{2}}{64 \ell(1+\ell)(-1+2 \ell)(1+2 \ell)(3+2 \ell) n^{5}}+\frac{9\left(-3-7 \ell+9 \ell^{2}+32 \ell^{3}+16 \ell^{4}\right)}{32 \ell^{2}(1+\ell)^{2}(-1+2 \ell)(1+2 \ell)^{2}(3+2 \ell) n^{4}}\right.} \\
& \left.+\frac{3\left(9+36 \ell-27 \ell^{2}-286 \ell^{3}-303 \ell^{4}+480 \ell^{5}+1280 \ell^{6}+960 \ell^{7}+240 \ell^{8}\right)}{32 \ell^{3}(1+\ell)^{3}(-1+2 \ell)^{2}(1+2 \ell)^{3}(3+2 \ell)^{2} n^{3}}\right]\langle\vec{L} \cdot \vec{S}\rangle \\
+ & {\left[\frac{1}{16(-1+2 \ell)(1+2 \ell)(3+2 \ell) n^{5}}-\frac{3\left(-2+3 \ell+3 \ell^{2}\right)}{16 \ell(1+\ell)(-1+2 \ell)(1+2 \ell)^{2}(3+2 \ell) n^{4}}\right.} \\
& \left.+\frac{-6-11 \ell+99 \ell^{2}+100 \ell^{3}-250 \ell^{4}-360 \ell^{5}-120 \ell^{6}}{16 \ell^{2}(1+\ell)^{2}(-1+2 \ell)^{2}(1+2 \ell)^{3}(3+2 \ell)^{2} n^{3}}\right] \vec{S}^{2} \\
+ & {\left[-\frac{69}{512 n^{6}}+\frac{-17+20 \ell+20 \ell^{2}}{8(-1+2 \ell)(1+2 \ell)(3+2 \ell) n^{5}}-\frac{3}{4(1+2 \ell)^{2} n^{4}}\right.} \\
& \left.\left.+\frac{3+48 \ell+64 \ell^{2}+32 \ell^{3}+16 \ell^{4}}{16 \ell(1+\ell)(-1+2 \ell)(1+2 \ell)^{3}(3+2 \ell) n^{3}}\right]\right\} .
\end{aligned}
$$

This expression agrees with previous results for $\ell=1[9,10]$, and is consistent with the coefficients of $\xi(j, \ell, s), \vec{S}^{2}$, and 1 that can be obtained from the results of [10]. Our value for the coefficient of $\langle\vec{L} \cdot \vec{S}\rangle$ differs from that of [10] when $\ell>1$ [44].

There results include only recoil corrections. Also appearing at $O\left(m \alpha^{6}\right)$ are corrections coming from higher order terms in the matching coefficients, which represent high-energy contributions in various sub-diagrams. Some of the matching coefficients can be derived from knowledge of the usual QED Dirac and Pauli form factors, as in [34, 35], while others require special calculation, such as the "radiative-recoil" corrections [8] and the three-photon-exchange contributions to the contact term (Fig. 1i) [9, 39-41]. Other corrections that contribute to S states come from one-, two-, and three-photon annihilation as tabulated in [8].

\section{Prospects for future work}

We believe that we have the tools in place to complete the calculation of all $O\left(m \alpha^{7}\right)$ corrections to positronium energy levels. Dimensionally regularized NRQED along with the NRQED Bethe-Salpeter equation and the method of regions provides a powerful scheme for identifying all contributions at the desired order and bringing them into a convenient form for evaluation. These methods have been tested by using them to complete successful re-evaluations of all contributions at orders $m \alpha^{4}$ and $m \alpha^{5}$ and the recoil corrections at $O\left(m \alpha^{6}\right)$. Completion of the $O\left(m \alpha^{7}\right)$ calculation will require a number of new NRQED calculations, the evaluation of additional hard contributions such as those coming from four-photon-exchange and four-photon-annihilation graphs, and a significant amount of organization to pull together terms already known (such as those discussed in [45]). 


\section{Acknowledgments}

We are extremely grateful to Jacek Zatorski for help in tracking down the differences in our results. We acknowledge useful conversations with Krzysztof Pachucki, Savely Karshenboim, and Michael Eides, and the support of the National Science Foundation through Grant No. PHY1707489 and of the Franklin \& Marshall College Hackman Scholars Program.

\section{References}

[1] M. Deutsch, Evidence for the formation of positronium in gases, Phys. Rev. 82, 455-456 (1951).

[2] S. DeBenedetti and H. C. Corben, Positronium, Ann. Rev. of Nucl. Sci. 4, 191-218 (1954).

[3] V. W. Hughes, Positronium and muonium, in proceedings of Physik 1973, Plenarvorträge Physikertagung, 37th, pp. 123-155 (1973).

[4] B. Maglich, Discovery of positronium, in Adventures in Expt. Phys. 4, 63-127 (1973).

[5] S. Berko and H. N. Pendleton, Positronium, Ann. Rev. Nucl. Part. Sci. 30, 543-581 (1980).

[6] A. P. Mills, Jr. and S. Chu, Precision measurements in positronium, in Quantum Electrodynamics, ed. by T. Kinoshita, pp. 774-821 (1990).

[7] S. G. Karshenboim, Precision study of positronium: testing bound state QED theory, Internat. J. Mod. Phys. A 19, 3879-3896 (2004).

[8] K. Pachucki and S. G. Karshenboim, Complete results for positronium energy levels at order $m \alpha^{6}$, Phys. Rev. Lett. 80, 2101-2014 (1998).

[9] A. Czarnecki, K. Melnikov, and A. Yelkhovsky, Positronium S-state spectrum: Analytic results at $O\left(m \alpha^{6}\right)$, Phys. Rev. A 59, 4316-4330 (1999).

[10] J. Zatorski, $O\left(m \alpha^{6}\right)$ corrections to energy levels of positronium with nonvanishing orbital angular momentum, Phys. Rev. A 78, 032103 (2008).

[11] The results of [10] must be corrected at one point as discussed in Sect. 3.

[12] S. G. Karshenboim, New logarithmic contributions in muonium and positronium, JETP 76, 541-546 (1993), [Zh. Eksp. Teor. Fiz. 103, 1105-1117 (1993)].

[13] K. Melnikov and A. Yelkhovsky, $O\left(m \alpha^{7} \ln ^{2} \alpha\right)$ corrections to positronium energy levels, Phys. Lett. B 458, 143-151 (1999).

[14] K. Pachucki and S. G. Karshenboim, Higher-order recoil corrections to energy levels of two-body systems, Phys. Rev. A 60, 2792-2798 (1999).

[15] B. A. Kniehl and A. A. Penin, Order $\alpha^{7} \ln (1 / \alpha)$ contribution to positronium hyperfine splitting, Phys. Rev. Lett. 85, 5094-5097 (2000).

[16] K. Melnikov and A. Yelkhovsky, $O\left(\alpha^{3} \ln \alpha\right)$ corrections to muonium and positronium hyperfine splitting, Phys. Rev. Lett. 86, 1498-1501 (2001).

[17] R. J. Hill, New value of $m_{\mu} / m_{e}$ from muonium hyperfine splitting, Phys. Rev. Lett. 86, 3280-3283 (2001).

[18] S. R. Marcu, Ultrasoft contribution to the positronium hyperfine splitting, Masters Thesis, University of Alberta, 2011. 
[19] G. S. Adkins and R. N. Fell, Positronium hyperfine splitting at order $m \alpha^{7}$ : Light-by-light scattering in the two-photon-exchange channel, Phys. Rev. A 89, 052518 (2014).

[20] M. I. Eides and V. A. Sheyuto, Hard nonlogarithmic corrections of order $\mathrm{m}^{7}$ to hyperfine splitting in positronium, Phys. Rev. D 89, 111301(R) (2014).

[21] M. I. Eides and V. A. Shelyuto, Hard three-loop corrections to hyperfine splitting in positronium and muonium, Phys. Rev. D 92, 013010 (2015).

[22] M. I. Eides and V. A. Shelyuto, Hard three-loop corrections to hyperfine splitting, Int. J. Mod. Phys. A 31, 1641030 (2016).

[23] M. I. Eides and V. A. Shelyuto, Hyperfine splitting in muonium and positronium, Int. J. Mod. Phys. A 31, 1645034 (2016).

[24] G. S. Adkins, M. Kim, C. Parsons, and R. N. Fell, Three-photon-annihilation contributions to positronium energies at order $m \alpha^{7}$, Phys. Rev. Lett. 115, 233401 (2015).

[25] G. S. Adkins, C. Parsons, M. D. Salinger, R. Wang, and R. N. Fell, Positronium energy levels at order $m \alpha^{7}$ : Light-by-light scattering in the two-photon-annihilation channel, Phys. Rev. A 90, 042502 (2014).

[26] G. S. Adkins, C. Parsons, M. D. Salinger, and R. Wang, Positronium energy levels at order $m \alpha^{7}$ : Vacuum polarization corrections in the two-photon-annihilation channel, Phys. Lett. B 747, 551-555 (2015).

[27] G. S. Adkins, L. M. Tran, and R. Wang, Positronium energy levels at order $\mathrm{m}^{7}$ : Product contributions in the two-photon-annihilation channel, Phys. Rev. A 93, 052511 (2016).

[28] M. I. Eides and V. A. Shelyuto, One more hard three-loop correction to parapositronium energy levels, Phys. Rev. D 96, 011301(R) (2017).

[29] M. Baker, P. Marquard, A. A. Penin, J. Piclum, and M. Steinhauser, Hyperfine splitting in positronium to $O\left(\alpha^{7} m_{e}\right)$ : One photon annihilation contribution, Phys. Rev. Lett. 112, 120407 (2014).

[30] G. S. Adkins, Higher order corrections to positronium energy levels, J. Phys.: Conf. Series 1138 , 012005 (2018).

[31] R. A. Ferrell, The positronium fine structure, Phys. Rev. 84, 858-859 (1951).

[32] A. Pineda and J. Soto, Potential NRQED: The positronium case, Phys. Rev. D 59, 016005 (1998).

[33] W. E. Caswell and G. P. Lepage, Effective Lagrangians for bound state problems in QED, QCD, and other field theories, Phys. Lett. B 167, 437-442 (1986).

[34] T. Kinoshita and M. Nio, Radiative corrections to the muonium hyperfine structure: The $\alpha^{2}(Z \alpha)$ correction, Phys. Rev. D 53, 4909-4929 (1996).

[35] R. J. Hill, G. Lee, G. Paz, and M. P. Solon, NRQED Lagrangian at order $1 / M^{4}$, Phys. Rev. D 87, 053017 (2013).

[36] G. S. Adkins, The hydrogen atom in $D=3-2 \varepsilon$ dimensions, Phys. Lett. A 382, 1545-1549 (2018).

[37] G. S. Adkins, M. F. Alam, C. Larison, and R. Sun, Coulomb expectation values in D= 3 and $D=3-2 \varepsilon$ dimensions, arXiv:1908.02324 (2019).

[38] M. Beneke and V. A. Smirnov, Asymptotic expansion of Feynman integrals near threshold, Nucl. Phys. B 522, 321-344 (1998). 
[39] K. Pachucki, Effective Hamiltonian approach to the bound state: Positronium hyperfine structure, Phys. Rev. A 56, 297-304 (1997).

[40] A. Czarnecki, K. Melnikov, and A. Yelkhovsky, Positronium hyperfine splitting: Analytical value at $O\left(m \alpha^{6}\right)$, Phys. Rev. Lett. 82, 311-314 (1999).

[41] K. Pachucki, Recoil effects in positronium energy levels to order $\alpha^{6}$, Phys. Rev. Lett. 79, 4120-4123 (1997).

[42] A. V. Smirnov, FIRE5: A C++ implementation of Feynman Integral REduction, Comput. Phys. Comm. 189, 182-191 (2015).

[43] B. R. Johnson and J. O. Hirschfelder, The radial reduced Coulomb Green's function, J. Math. Phys. 20, 2484-2501 (1979).

[44] J. Zatorski, private communication (2019). The difference appears to be due to a small error in Zatorski's code.

[45] M. I. Eides, H. Grotch, and V. A. Shelyuto, Theory of light hydrogenic bound states, Springer Tracts in Modern Physics, Volumn 222, Berlin, 2007. 